\title{
Rebuttal to "Causal effect of vitamin D on prostate cancer using Mendelian randomization approach"
}

\author{
Olivia Trummer ${ }^{1} \cdot$ Wilfried Renner $^{2} \cdot$ Tanja Langsenlehner $^{3}$
}

Received: 26 August 2015 / Accepted: 31 August 2015 / Published online: 21 September 2015

(C) Springer-Verlag Berlin Heidelberg 2015

Dear Editor,

In their letter, Haining et al. [1] addressed potential limitations of our study, such as the lack of plasma vitamin D levels or the moderate statistical power. We are aware of these potential limitations and agree that larger studies including plasma vitamin D measurements may provide further insight whether vitamin D plays a causal role in prostate cancer outcome.

\section{Compliance with ethical standards}

Conflict of interest The authors declare that they have no conflict of interest.

Ethical standards This study was carried out in accordance with the principles of the Declaration of Helsinki of 1975, as revised in 2008.

\section{Reference}

1. Yu H, Xu P, Cui Y (2015) Causal effect of vitamin D on prostate cancer using Mendelian randomization approach. World J Urol. doi:10.1007/s00345-015-1672-7

This reply refers to the article available at doi:10.1007/s00345-015-1672-7.

Wilfried Renner

wilfried.renner@medunigraz.at

1 Division of Endocrinology and Metabolism, Department of Internal Medicine, Medical University of Graz, Graz, Austria

2 Clinical Institute of Medical and Chemical Laboratory Diagnostics, Medical University of Graz, 8036 Graz, Austria

3 Department of Therapeutic Radiology and Oncology, Medical University of Graz, Graz, Austria 